\title{
Controlling Laser Material Processing with Real-Time Algorithms on Cellular Neural Networks
}

Strohm, Peter ${ }^{1}$; Krause, Olaf ${ }^{2}$; Blug, Andreas ${ }^{1}$; Carl, Daniel ${ }^{1}$; Panzner, Michael ${ }^{2}$; Höfler, Heinrich ${ }^{1}$

1: Fraunhofer-Institut für Physikalische Messtechnik IPM, Heidenhofstr. 8, 79110 Freiburg, Germany

2: Fraunhofer-Institut für Werkstoff- und Strahltechnik IWS, Winterbergstr. 28, 01277 Dresden, Germany

\begin{abstract}
Today image processing using a coaxial camera setup is used to monitor the quality of laser material processes such as laser welding, cutting, ablation or scribing. For real-time control of highly dynamic laser processes these systems are far to slow. This article proposes a sensing system for the next step: Using image based quality features in a real-time algorithm to form an instant feedback signal with up to $14 \mathrm{kHz}$ in order to maintain the process in the desired state.
\end{abstract}

The key component of the control system is a camera based on Cellular Neural Networks (CNN). This Single Instruction Multiple Data technology enables real time image processing by integrating processing units in every camera pixel. Moreover each of the pixel units is interconnected with its nearest neighbors which is optimal for most image processing algorithms. This article describes the CNN technology together with results obtained for $\mathrm{CNN}$-based feedback systems designed for the closed-loop control laser ablation and laser welding algorithms.

\section{Introduction}

Error prevention and correction is a key to fast and reliable laser processes from automobile production to micro mechanics. Closed loop control systems can help to achieve high productivity and constant quality even under changing process conditions or material properties. Camera based in-process control allows to employ algorithms for image processing which are widely independent of the exact process parameters, reducing the adaption effort for different applications compared to simple photo diode systems. In particular for laser welding and laser cutting, so called monitoring systems are used [1-3]. These systems acquire images during the process and perform a quality assessment by a statistical analysis of one or more image features.

However, these laser material processes occur very fast and therefore conventional image processing reaches its limits. Our strategy was to take such known features and perform a faster real time image processing on a novel camera technique, the so called Cellular Neural Networks (CNN). This article describes the CNN technology, the setup of closed loop control systems and results obtained for two applications. The first application is a laser ablation process where star-shaped areas within diamond-like carbon layers are ablated from a gold layer. Here, a prototype of a closed loop control system was developed within a cooperation between the Fraunhofer IPM and the Fraunhofer IWS. In the second process is a closed-loop control system for laser welding processes which was developed in cooperation with Fraunhofer IPM, the IFSW Institut für Strahlwerkzeuge at Stuttgart University and the Technical University of Dresden.

\section{Cellular Neural Networks (CNN)}

Conventional computing systems with the exception of FPGA are usually "Single Instruction Single Data" (SISD) architectures [4]. Here a program consists of a series of instructions which are applied sequentially to single data elements by one or few processor cores. Therefore the execution time lies in the order of $\mathrm{N}$ or $\mathrm{N}^{2}$, where $\mathrm{N}$ is the number of pixels. One alternative are "Single Instruction Multiple Data" (SIMD) computing architectures in which each instruction of a program is applied in parallel to a large number of data. Such SIMD applications are especially efficient for image processing applications which frequently apply the same operation on different pixels of the image. On SIMD systems, these operations can be executed simultaneously in a single step. Cellular neural networks, which were originally invented by Chua and Yang [5], can be considered as a technology which enables computing elements and storage cells to be integrated into camera pixels. It provides a possible basis for the integration of SIMD architectures into the electronic circuitry of the pixels in CMOS cameras. The CNN universal machine forms the basis [6]. It can be implemented in a small space with the aid of CMOS processes in an array of optical sensors together with analogue and digital data processing elements 


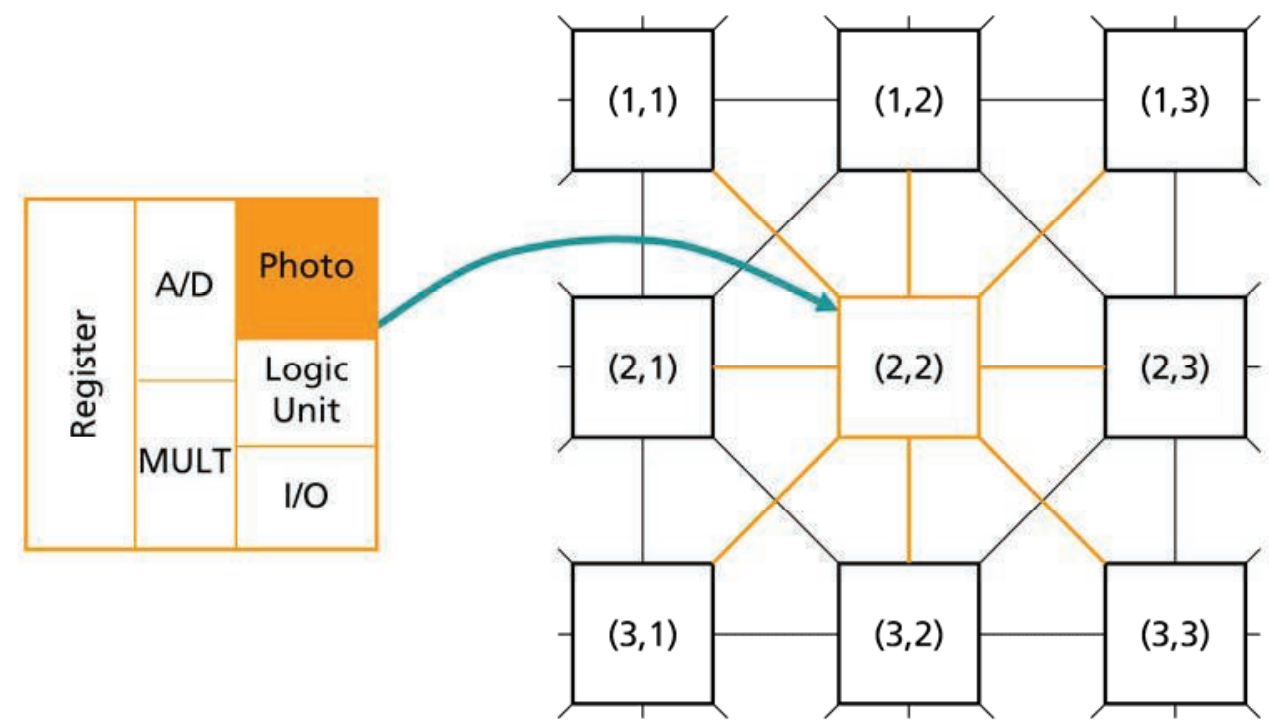

Fig. 1: CNN enable the integration of processor elements in the electronic circuitry of CMOS cameras.

$[7,8]$. Such systems are spatial arrangements of locally connected, non-linear dynamic units that are usually called cells. In addition to the photo sensor, each of these cells contains storage and computing elements as well as switching elements for the input/output of results (Fig. 1). In this way, cellular neural networks link the intensity values of spatially and temporally adjacent pixels and they are especially efficient for operations exploiting these neighborhoods. Typical examples for neighborhood based operations are morphological and logical operations as we use for the detection of the full penetration hole.

\section{CNN-Based Closed-Loop Control System}

To build a closed loop control system, one needs a pair of a controlled variable, i.e. an image feature, representing the state of the system and of a feedback parameter which influences the controlled variable on a suitable time scale. As described above, within a CNN camera, every pixel has its own processor. This means, that the number of results equals to the number if pixels, i.e. the result is an image. Therefore, in a closed-loop control system, a decision making element is required which actuates the feedback signal S. For this reason, the EyeRIS-system developed by Anafocus [9] was used to execute the algorithms for the detection of the full penetration hole. It consists of a CNN based camera chip called Q-Eye with $176 \times 144$ cells and of an additional FPGA based NIOS II processor by Altera to control the operation of the whole vision system and to analyze the information output of the Q-Eye, e.g. performing all the decision making and actuation tasks.

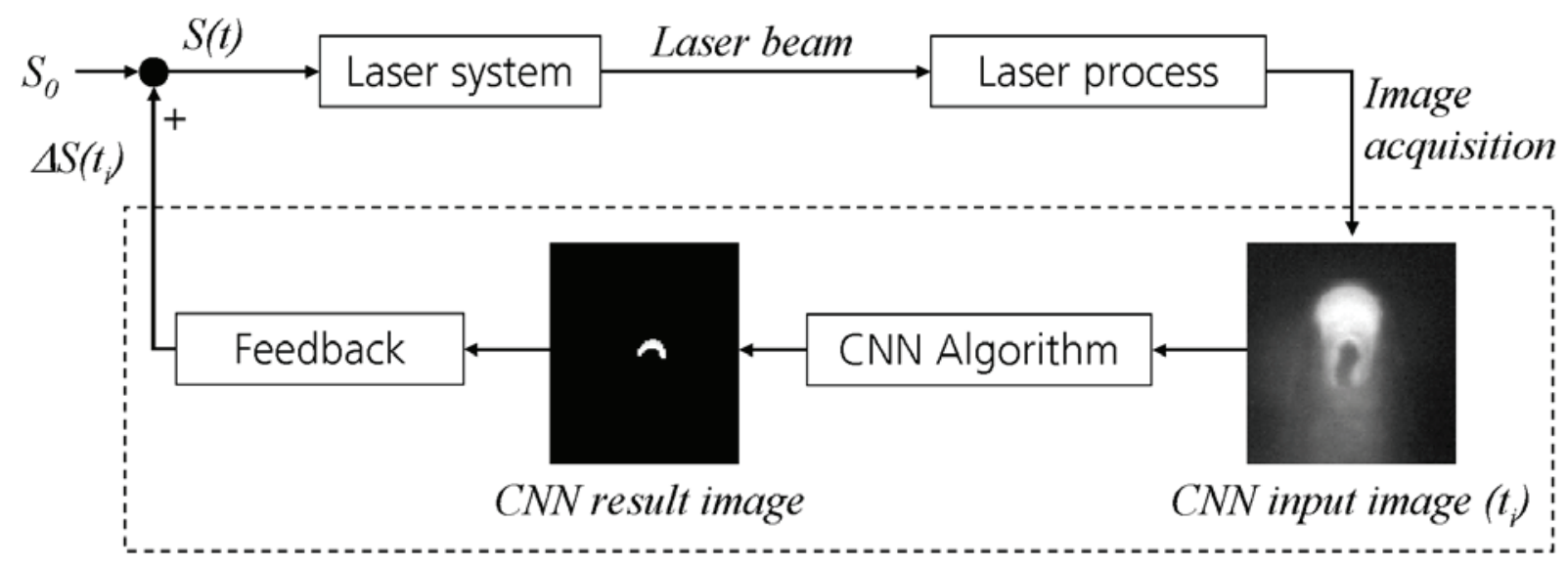

Fig. 2: Sketch of the closed-loop control systems. The dashed line marks those parts executed within the CNN camera. 


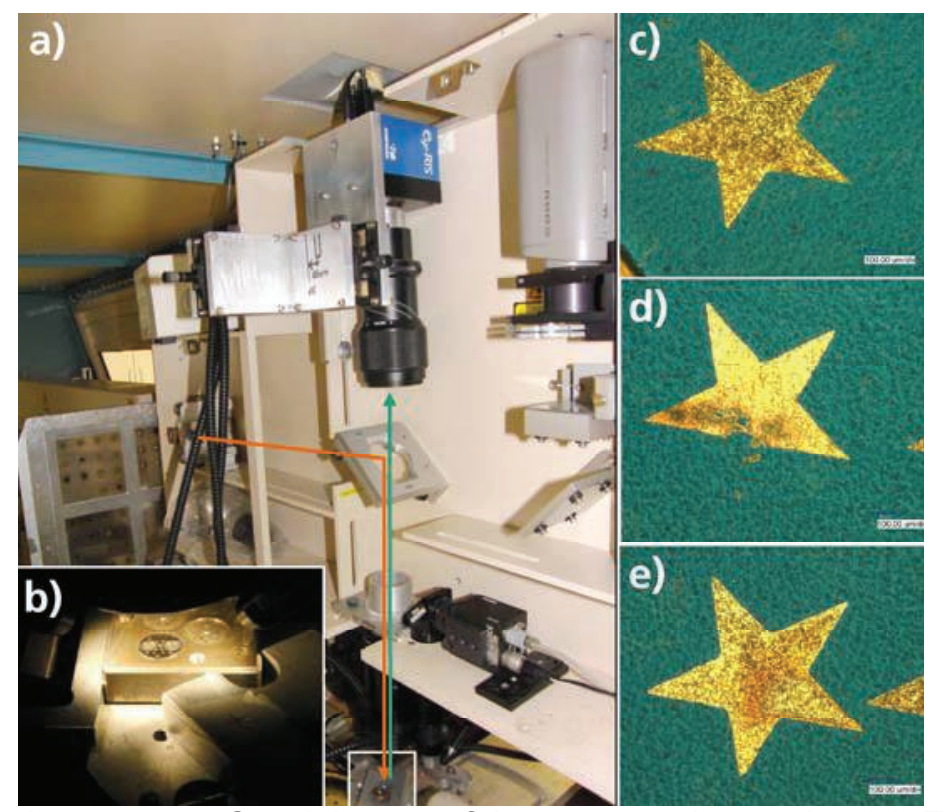

Fig. 3: Laser ablation process. Coaxial setup of the process observation (a). The orange arrow marks the path of the excimer laser beam, the green one the optical path of the CNN camera. b):

Enlarged image of the target. Right side: Microscope images of a good' star with homogeneous surface quality (c), a star with incomplete ablation (d) and with a bad surface quality (e).

\section{Closed-loop Control of a Laser Ablation Process}

The investigated ablation process is based on an excimer laser with a pulse energy of $320 \mathrm{~mJ}$, a repetition rate of $150 \mathrm{~Hz}$, and a pulse duration of $25 \mathrm{~ns}$. The aim of the process is to remove star-shaped areas from a golden disk coated with a diamond-like carbon layer. Figure 3 shows the measurement setup. In figure 3a) the masked laser beam comes from the left and is directed to the target over a dicroidic mirror. The CNN camera is mounted coaxially to the laser beam. As shown in figure $3 \mathrm{~b}$ ), the target is illuminated by three Xenon lamps. The problem of this process is that the number of pulses needed for a good surface quality of the stars varies due to inhomogeneous material properties. If the number of pulses is too low, the shape of the stars is incomplete as shown in figure 3d), if it is too high, the surface quality of the gold layer deteriorates due to the surface morphology.

In the case of the ablation process, the input image is evaluated for the shape and the brightness of the stars. The feedback signal $S(t)$ is simply a start/stop signal. Figure 4 sketches the $C N N$ algorithm. Image a) shows the region of interest. The image of the first laser pulse (figure $4 \mathrm{~b}$ ) is used to generate a mask for the process (figure 4c). After the mask generation, laser pulses are generated until a certain percentage of the mask area, e.g. $85 \%$, exceeds a certain threshold or until the shape of the star does not change anymore. The images d) and e) show images of intermediate and final results. During the test processes, the number of pulses necessary for a homogeneous quality varied between 76 and 158 images. As a result the control system the average number of pulses decreases. Therefore, time and energy consumption of the process are reduced and at the same time the surface quality of the final product is improved.
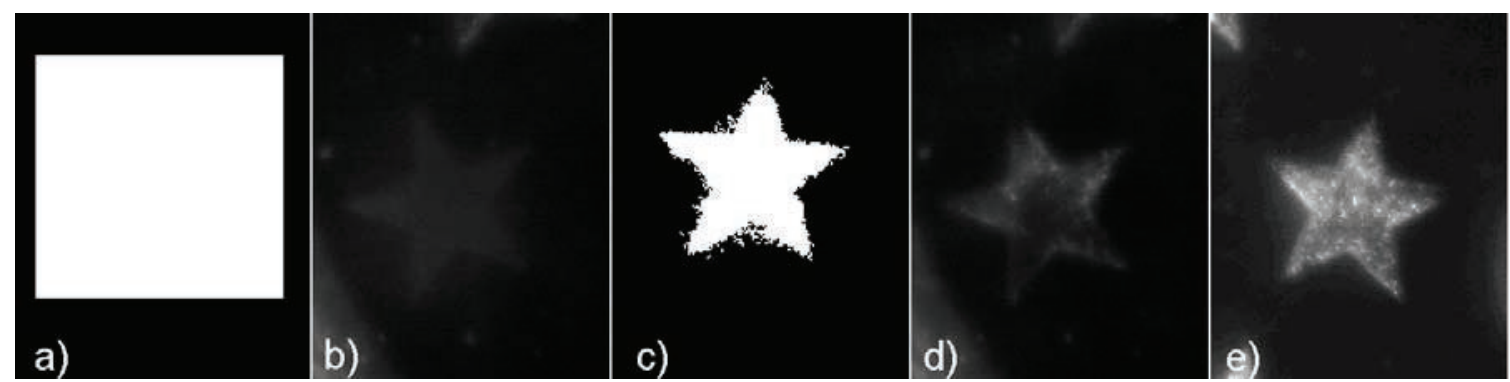

Fig. 4: CNN algorithm. a) Region of interest. b) Image obtained after the first pulse. c) Mask generated from image b). d) Intermediate result. e) Final result. 

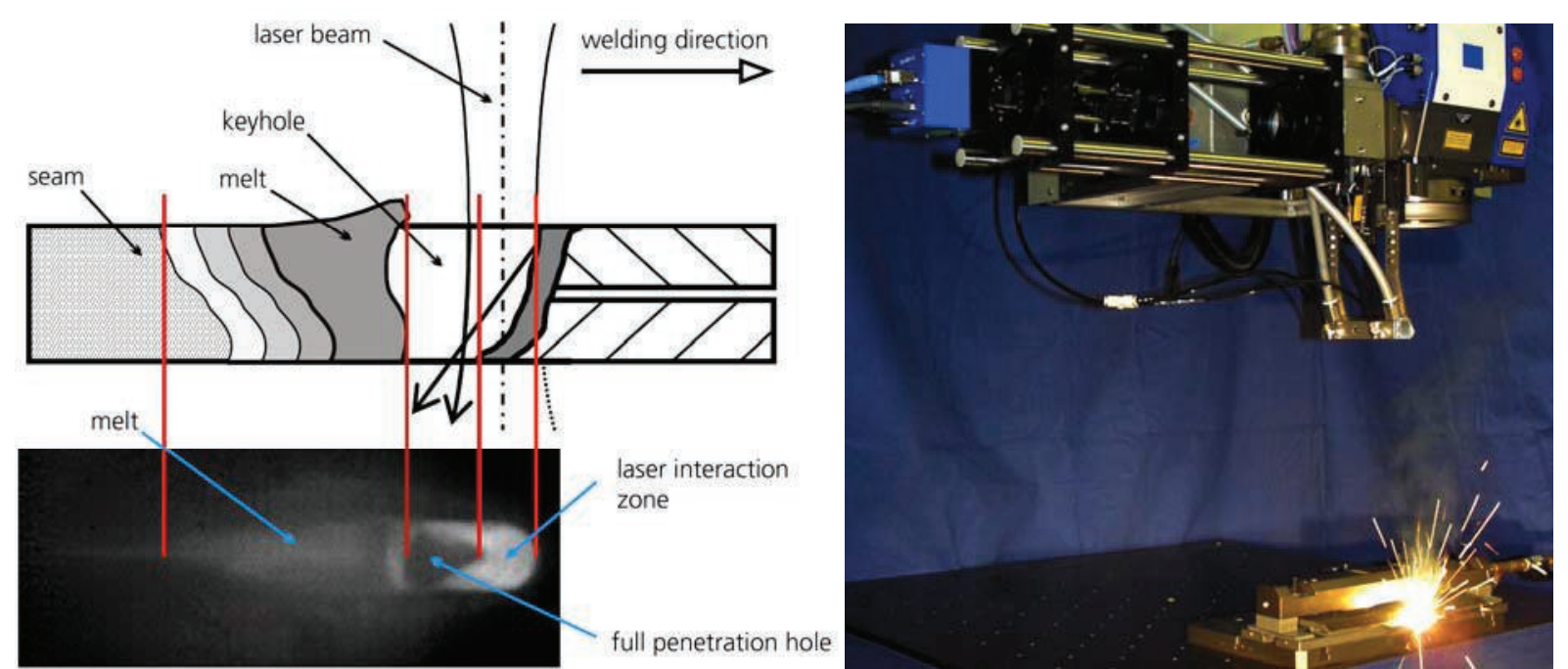

Fig. 5: Left: Cross section of an overlap-joint laser welding process and a picture of the interaction zone. The full penetration hole is a feature of the thermal image showing that the depth of the keyhole reaches the bottom of the lower sheet. Right: For the coaxial observation, the CNN camera (blue box, left) was mounted to the process window of the welding head.

\section{Closed-Ioop Control of Keyhole Laser Welding Processes}

Laser-beam welding has evolved into a popular jointing method because it combines a high bonding quality with a high speed. A laser beam with a high intensity is focused onto the work piece. In so called deep penetration welding processes, the surface of the work piece is heated up to vapor temperature and Within the focus of the laser very high temperatures occur that cause the material to melt. During this time a capillary filled with metal vapor develops forming the so called keyhole (see figure 5, left). When the keyhole extends to the bottom of the work piece, the capillary opens. In this case, the so called full penetration hole is observed as a dark spot in the spectral range of about 900 to $1000 \mathrm{~nm}$. Thus, the full penetration hole is an image feature indicating that the depth of the welding process equals the thickness of the sheets.

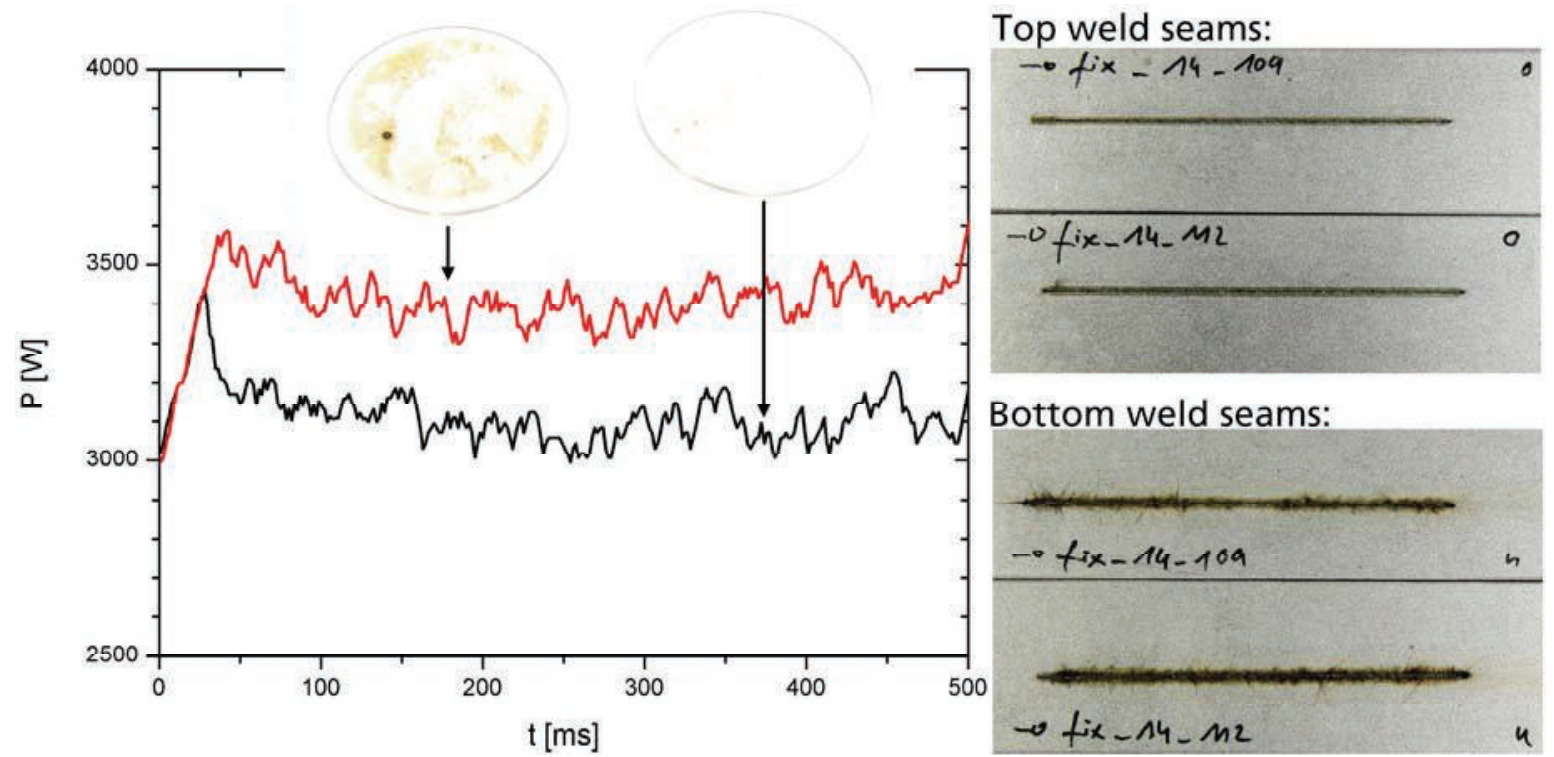

Fig. 6: Example for a process drift compensated by the closed loop control system: Sample fix_14_109 was welded with an almost clean protection window (black curve), sample fix_14_112 with

a dirty one (red curve). The laser power is increased automatically from 3100 to $3400 \bar{W}$ without visible change in the weld seams (right side). The focal diameter was $0.2 \mathrm{~mm}$, sheet thickness $1 \mathrm{~mm}$, gap size $0.1 \mathrm{~mm}$, and feeding rate $9 \mathrm{~m} / \mathrm{min}$. 

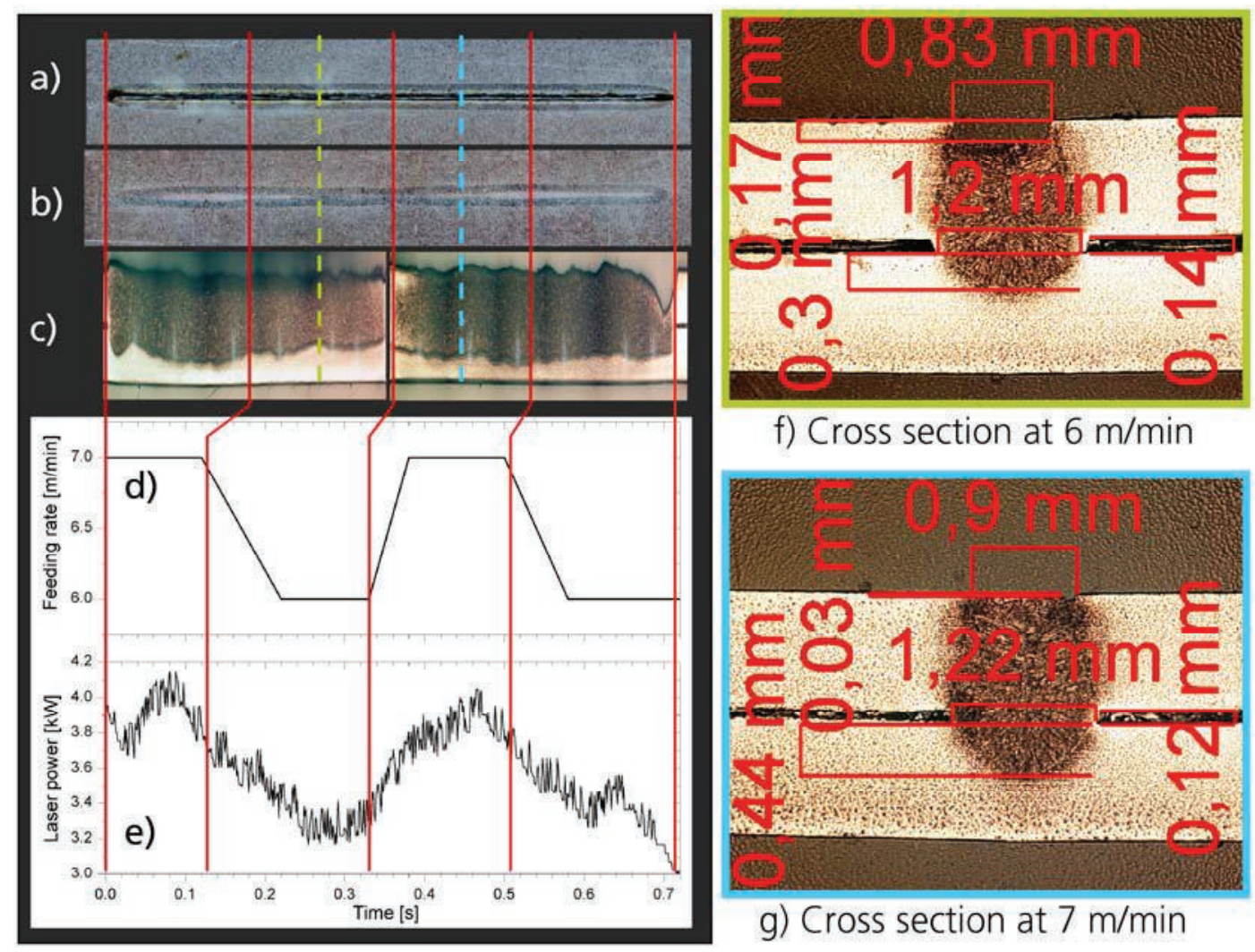

f) Cross section at $6 \mathrm{~m} / \mathrm{min}$

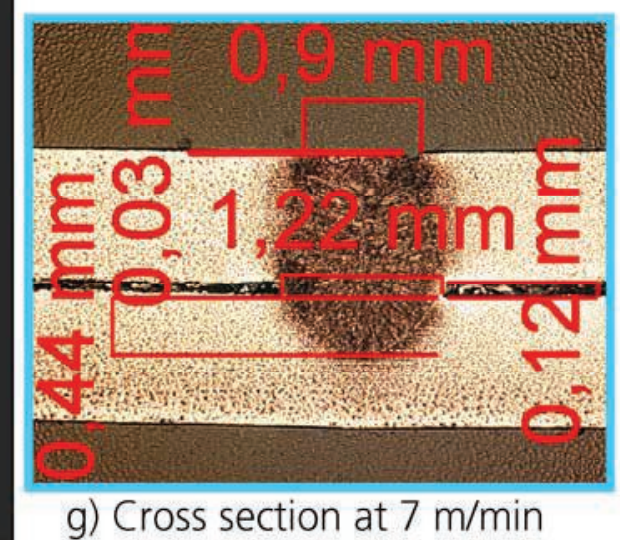

Fig. 7: Compensation of variations in the feeding rate at the partial penetration state: Top welding seam (a), heat trace at the bottom sheet (b), longitudinal cross section (c), feeding rate varying between 7 and $6 \mathrm{~m} / \mathrm{min}$ (d), automatically adapted laser signal (e) and cross sections at the green dashed line at a feeding rate of $6 \mathrm{~m} / \mathrm{min}(\mathrm{f})$ and at $7 \mathrm{~m} / \mathrm{min}(\mathrm{g})$. The focal diameter was $0.6 \mathrm{~mm}$, the material was zinc coated steel with a thickness of $1 \mathrm{~mm}$, gap size was $0.1 \mathrm{~mm}$.

In the case of the laser welding process, the thermal image is evaluated by the CNN camera for the contour of the full penetration hole and the feedback signal $S(\mathrm{t})$ is the laser power. In this way, the welding process is guided at the minimum laser power necessary for full penetration. The high frame rate of up to $14 \mathrm{kHz}$ of the evaluation of the CNN camera is necessary because the full penetration hole fluctuates in the $\mathrm{kHz}$ range [10]. The result is a welding process where the laser power is adapted automatically to the current state of the process. Typical process drifts occurring during production such as polluted protection windows (figure 6), variations of the focal position or of the feeding rate at robot welding are compensated.

The system works in a large variety of material and laser configurations. It is stable under varying process conditions such as steps in feeding rates or material thickness. It works with zinc coated steel as well as with aluminum [11]. As figure 7 shows, it works not only at the full penetration state but also at partial penetration. In this state, the bottom of the keyhole crosses the gap between the lower and the upper sheet at an overlap joint. Due to the interrupted heat flux between the two sheet, the upper surface of the bottom sheet remains colder than the walls of the vapor capillary and an image feature very similar to the full penetration hole appears [12].

\section{Conclusion}

This article describes two laser material processes for which quality control or even closed-loop control based on image processing are difficult or even impossible with conventional camera systems. Therefore, a novel camera technique based on cellular neural networks (CNN) was introduced. This technology enables the integration of processing elements into the electronic circuitry of each camera pixel. The result is a Single Instruction Multiple Data (SIMD) computing architecture on the camera chip which allows for real time image processing at frame rates of up to $14 \mathrm{kHz}$.

This technique was applied to two laser material processes. The first one was a masked laser ablation process. There, the effect of each laser pulse onto the shape and brightness of the inscribed stars was 
controlled by the CNN camera. As a result the control system the average number of pulses decreases. Therefore, time and energy consumption of the process is reduced and at the same time the surface quality of the final product is improved.

The second application was a so called deep penetration laser welding process where the full penetration hole appears at two welding depths: At the state of full penetration and at partial penetration when the bottom of the capillary crosses the gap between the sheets of an overlap joint. In both cases process drifts due to a polluted process window or variations in the focal position or in the feeding rate are compensated because the process is always guided at the minimum required laser power. In particular at car body manufacturing, the partial penetration prevents the evaporation of zinc at the bottom weld seam which reduces the corrosion in these areas.

\section{Acknowledgement}

This work was funded by the Baden-Württemberg Stiftung gGmbH within the project "Analoge Bildverarbeitung mit zellularen neuronalen Netzen (CNN) zur Regelung laserbasierter Schweißprozesse (ACES)".

\section{References}

[1] S. Kaierle, P. Abels G. Kapper C. Kratzsch J. Michel W. Schulz R. Poprawe (2001): State of the Art and New Advances in Process Control for Laser Materials Processing. Paper 805. In: Congress proceedings. ICALEO, Orlando, Fla: LIA Laser Inst. of America.

[2] Müller-Borhanian, Jürgen (2005): Integration optischer Messmethoden zur Prozesskontrolle beim Laserstrahlschweissen (INESS). Abschlussbericht zum Verbundprojekt. München: Utz (Forschungs- und Tagungsberichte).

[3] Fabrice Bardin, Adolfo Cobo, Jose M. Lopez-Higuera, Olivier Collin, Pascal Aubry (2005): Closed-loop power and focus control of laser welding for full-penetration monitoring. In: APPLIED OPTICS (Vol. 44, No. 1, January $\left.1^{\text {st }}, 2005\right)$, pp. 13-21.

[4] Flynn, M.: Some Computer Organizations and Their Effectiveness, IEEE Trans. Comput., Vol. C21 (1972), pp. 948

[5] L. O. Chua, L. Yang, "Cellular Neural Networks: Theory", IEEE TRANSACTIONS ON CIRCUITS AND SYSTEMS, VOL. 35, NO. 10, OCT. 1988

[6] T. Roska and L.O. Chua, "The CNN Universal Machine: an analogic array computer", IEEE Transactions on Circuits and Systems-II Vol.40, pp. 163-173, March, 1993

[7] Rodríguez-Vázquez, A., Liñán-Cembrano, G., Carranza, L., Roca-Moreno, E., Carmona-Galán, R., Jiménez-Garrido, F., Domínguez-Castro, R., Espejo Meana, S.: ACE16k: The Third Generation of Mixed-Signal SIMD-CNN ACE Chips Toward VSoCs. IEEE TRANSACTIONS ON CIRCUITS AND SYSTEMS—I: REGULAR PAPERS, VOL. 51, NO. 5, pp. 851, MAY 2004

[8] P. Dudek, P.J. Hicks, "A general-purpose processor per-pixel analog SIMD vision chip", IEEE Transactions on Circuits and Systems I: Regular Papers, Volume 52, Issue 1, pp. 13 - 20, Jan. 2005.

[9] Company Anafocus, Avd. Isaac Newton s/n, Pabellón de Italia, Ático Parque Tecnológico Isla de la Cartuja, 41092 Sevilla, Spain

[10] A. Blug, F. Abt, L. Nicolosi, D. Carl, F. Dausinger, H. Höfler, R. Tetzlaff, R. Weber: CLOSED LOOP CONTROL OF LASER WELDING PROCESSES USING CELLULAR NEURAL NETWORKCAMERAS: MEASUREMENT TECHNOLOGY. Paper 1504, Proc. of 28th International Congress on Applications of Lasers \& Electro-Optics (ICALEO 2009), Orlando, USA

[11] A. Blug, D. Carl, H. Höfler, F. Abt, A. Heider, R. Weber, L. Nicolosi, R. Tetzlaff: Closed-loop control of laser power using the full penetration hole image feature in aluminum welding processes. Proc. of LiM - Lasers in Manufacturing 2011 (LiM 2011), May 23rd - 26th, Munich

[12] F. Abt, A. Heider, R. Weber, A. Blug, D. Carl, H. Höfler, L. Nicolosi, R. Tetzlaff: Camera based closed loop control for partial penetration welding of overlap joints. Proc. of LiM - Lasers in Manufacturing 2011 (LiM 2011), May 23rd - 26th, Munich, Germany 